\title{
Symmetry Analysis of Turbulent Flows on the Base of the Variational Principles of Non-Equilibrium Thermodynamics and Theory of Q1D Systems
}

\author{
Cs. Mészáros, Á. Bálint
}

\begin{abstract}
A novel-type symmetry analysis of the basic mathematical formalism of some fundamental features of the convection-diffusion and turbulent flows is given on the base of the Riccati-type ordinary and matrix differential equation. Common symmetry features of the turbulent flow velocity and incommensurately modulated crystals are also briefly discussed.
\end{abstract}

\section{Introduction}

The mathematical modelling of the convection-diffusion processes even nowadays plays an important role in transport engineering problems, whose complexity is reflected in the nonlinear character of the ordinary differential equations (ODEs) and partial differential equations (PDEs) to be solved. The relevant basic PDE is:

$$
\frac{\partial c}{\partial t}-\nabla \cdot(D(c) \nabla c)-\frac{d K}{d c} \cdot \frac{\partial c}{\partial z}=0
$$

where $c=c(\vec{r}, t)$ denotes the concentration distribution function, $D=D(c, T, \ldots)$ is the diffusion coefficient, $K=K(c)$ is the hydraulic conductivity coefficient and z-axis corresponds to the direction of the gravitational acceleration. Using D'Alembert-type variables $\zeta$, the general form of the solution will be e.g. (Mszros et al., 2012):

$$
c(\zeta)=a_{0}+\sum_{i=1}^{q}\left(a_{i} \omega^{i}+b_{i} \omega^{-i}\right), a_{0}, a_{i}, b_{i}=\text { const } .
$$

where $q \in \mathfrak{R}^{+}$and the component solution functions obey the Riccati-type ODE $\frac{d \omega}{d \zeta}=k+$ $\omega^{2}$, with a parameter $k$ " depending on the actual experimental conditions.

Manuscript received December 24, 2014. ; accepted May 21, 2015.

Cs. Mészáros is with the Szent István University, Department of Physics and Process Control Gödölló, H-2100, Hungary; Á. Bálint is with the Óbuda University, Institute of Environmental Engineering Budapest, H-1034, Hungary 
Another well-known example is related (Landau and Lifshitz, 2000,a) e.g. to gravity waves on the free fluid surfaces resulting in the linearized form of the Riccati-type ODE $\frac{d^{2} f}{d z^{2}}-k^{2} f=0$ on the base of use of a usual rectangular coordinate-system and assumption, that the continuous elastic medium fills the infinite half-space $\mathrm{z}<0$, and the travelling waves propagating into $\mathrm{x}$-direction can be described as $u=f(z) \cdot e^{i(k x-\omega t)}$.

The relevance of the Riccati's ODE for describing of some basic features of the turbulent can be demonstrated within frame of the classical Landau-Hopf theory, too e.g. (Landau and Lifshitz, 2000, a). Namely, after decomposition of the velocity function to stationary velocity field $\vec{v}_{0}(\vec{r})$ and non-stationary velocity contribution $\vec{v}_{1}(\vec{r}, t)$, the latter will be determined by the PDE system:

$$
\frac{\partial \vec{v}_{1}}{\partial t}+\left(\vec{v}_{0} \cdot \nabla\right) \vec{v}_{1}+\left(\vec{v}_{1} \cdot \nabla\right) \vec{v}_{0}=-\frac{\nabla p_{1}}{\rho}+\frac{\eta}{\rho} \nabla^{2} \vec{v}_{1}, \nabla \cdot \vec{v}_{1}=0,
$$

with solution $\vec{v}_{1}(\vec{r}, t)=\vec{f}(\vec{r}) \cdot A(t) \equiv \vec{f}(\vec{r}) \cdot e^{\gamma_{1} t} \cdot e^{-i \omega_{1} t}$, where the amplitude function obeys relationship $\frac{d}{d t}|A|^{2}=2 \gamma_{1}|A|^{2}-\alpha|A|^{4}$. Although rarely emphasized, this equation is also of Riccati-type. Then, the general solution form (with $n$ non-stationary components in general case) of the velocity field function is:

$$
\vec{v}(\vec{r}, t)=\sum_{p_{1}} \ldots \sum_{p_{n}} \vec{A}_{p_{1} \ldots p_{n}}(\vec{r}) \cdot e^{-i \sum_{j=1}^{n} p_{j} \varphi_{j}}
$$

which will be of crucial importance at explaining the essential new result of the present study.

Therefore, extensive use of the Riccati-type ODE in continuum mechanics has its roots in the previously discussed classical results. This aspect has not been emphasized adequately in the literature.

\section{Application of the Riccati-type ordinary differential equation}

First of all, we recall here a basic invariance property of the Riccati-type ODE $y^{\prime}+f(x) y^{2}+$ $g(x) y+h(x)=0$, whose general solution is invariant (Mitrinović, 1983) with respect to the homographic transformation $y=\frac{Q(x) z+R(x)}{S(x) z+T(x)}, \quad(Q T-R S \neq 0)$. In order to solve the Riccati - ODE related to (2) we apply a more general form of it's, i.e.:

$$
y^{\prime}-y^{2}-\sum_{m} a_{m}(p) \zeta^{m}=0,
$$

where in functions $a_{n}=a_{n}(p)$ the independent variable is the percolation probability. If there is only one dominant term" in (5) (i.e. $y^{\prime}-y^{2}-a_{n}(p) \cdot \zeta^{n}=0$ ), the solution for $y(\zeta)$ is:

$$
\frac{C\left[\phi(\zeta) \cdot J_{\frac{n+3}{n+2}}\left(\frac{2 \phi(\zeta)}{n+2}\right)-J_{\frac{1}{n+2}}\left(\frac{2 \phi(\zeta)}{n+2}\right)\right]}{\zeta\left[C J_{\frac{1}{n+2}}\left(\frac{2 \phi(\zeta)}{n+2}\right)+Y_{\frac{1}{n+2}}\left(\frac{2 \phi(\zeta)}{n+2}\right)\right]}+\frac{\left[\phi(\zeta) \cdot Y_{\frac{n+3}{n+2}}\left(\frac{2 \phi(\zeta)}{n+2}\right)-Y_{\frac{1}{n+2}}\left(\frac{2 \phi(\zeta)}{n+2}\right)\right]}{\zeta\left[C J_{\frac{1}{n+2}}\left(\frac{2 \phi(\zeta)}{n+2}\right)+Y_{\frac{1}{n+2}}\left(\frac{2 \phi(\zeta)}{n+2}\right)\right]}
$$


(with $\phi(\zeta) \equiv \sqrt{a_{n}(p)} \cdot \zeta^{\frac{n}{2}+1}, C=$ const.) and $J_{k}(\zeta)$ and $Y_{k}(\zeta)$ denote the Bessel-functions of k-th order, which are of the first kind, and second kind respectively. It is obvious, that the basic invariance property of the Riccati ODE is reflected in the form of the solution (6), too.

The Navier-Stokes' equation may also be derived by variational calculus on the base of the "governing principle" (Gyarmati, 1970). The relevant Lagrangian density function is:

$$
\begin{aligned}
& L_{v \omega}^{*}=-\vec{v} \cdot(\rho \dot{\vec{v}}+\nabla p-\rho \vec{F})-\rho \Theta \vec{\omega} \cdot \dot{\vec{\omega}}-\frac{1}{2}\left(\eta_{v}-\frac{2}{3} \eta\right)(\nabla \cdot \vec{v})^{2}- \\
& -\eta\left[(\nabla \circ \vec{v})^{s}:(\nabla \circ \vec{v})^{s}\right]-\frac{\eta_{r}}{2}(\nabla \times \vec{v}-2 \vec{\omega})^{2},
\end{aligned}
$$

and the variation parameters $\Gamma_{i}(\mathrm{i}=1, \ldots \mathrm{f})$ are now intensive thermodynamic quantities. According to the basic principles of the variational calculus (Zelikin, 2001) at second-order variation procedures (generally: with several unknown functions), the $\hat{W}^{\prime}+\hat{Q}=\hat{W} \hat{R}^{-1} \hat{W}$ Riccati-type matrix equation must be solved with matrices $\hat{Q}$ and $\hat{R}$ built up from all possible types of second-order partial derivatives of the Lagrangian. Then, we arrive at:

$$
\hat{R} \equiv\left\|R_{i k}\right\|=\frac{1}{2}\left\|F_{y_{i}^{\prime} y_{k}^{\prime}}\right\|, \hat{Q} \equiv\left\|Q_{i k}\right\|=\frac{1}{2}\left(\left\|F_{y_{i} y_{k}}\right\|-\frac{d}{d x}\left\|F_{y_{i} y_{k}^{\prime}}\right\|\right)
$$

i.e., we are faced with solving of matrix equations with matrices having 5184 elements, and the block-structure of these rarely-filled matrices still represents an open problem.

\section{Analogies between dissipative structures and structural phase transitions leading to incommensurately modulated crystals}

Finally, we would like to call attention briefly on a novel possibility of studying phase transitions in crystals and creation of certain types of dissipative structures. Although this aspect had been emphasized in literature many times e.g. (Tribelskii, 1997), the application of symmetries quasi-one-dimensional $(Q 1 D)$ systems - the so called line groups e.g. (Vukovi et al., 1996) is still absent in this sense. In order to justify this statement, we summarize some classical results about this topic in the following table (the basic relationships about phase transitions giving incommensurate crystals are taken from Landau). Therefore, the incommensurate character is relevant for both turbulent flow (whose different harmonics have generally incommensurate frequencies) and modulated crystals, with two-, or more translationally invariant subsystems, having incommensurate elementary periods. 


\begin{tabular}{|l|l|l|}
\hline $\begin{array}{l}\text { Type of the } \\
\text { complex } \\
\text { system } \\
\text { undergoing } \\
\text { phase } \\
\text { transition }\end{array}$ & $\begin{array}{l}\text { Incommensurately } \\
\text { modulated crystal }\end{array}$ & Turbulent flow \\
\hline $\begin{array}{l}\text { Control } \\
\text { parameter }\end{array}$ & Temperature (T) & Reynolds number (Re) \\
\hline $\begin{array}{l}\text { Characteristi } \\
\text { c field type }\end{array}$ & $\begin{array}{l}\text { Electron-, spin } \\
\text { density function } \\
(\rho(\vec{r}), \vec{S}(\vec{r}))\end{array}$ & Velocity field $(\vec{v}(\vec{r}, t))$ \\
\hline $\begin{array}{l}\text { Order } \\
\text { parameter } \\
\text { function }\end{array}$ & $\rho(\vec{r})=\rho_{0}(\vec{r})+\sum_{\Lambda, v} \eta_{\lambda} \varphi_{\lambda}^{(\hat{y})}(\vec{r})$ & $\vec{v}(\vec{r}, t)=\sum_{p_{1}} \ldots \sum_{p_{n}} \vec{A}_{p_{1} \ldots p_{n}}(\vec{r}) \cdot e^{-i \sum_{j=1}^{n} p_{\phi_{j}}}$ \\
\hline $\begin{array}{l}\text { Behaviour in } \\
\text { the vicinity } \\
\text { of the critical } \\
\text { point and } \\
\text { Lifshitz, } \\
\text { 2000, b)): }\end{array}$ & $\eta \sim \sqrt{T-T_{c r i t .}}$ & $|A|_{\max } \sim \sqrt{\operatorname{Re}-\operatorname{Re}_{c r i t .}}$ \\
\hline
\end{tabular}

\section{Conclusions}

In the present work an attempt is given for a general symmetry treatment of the convectiondiffusion and turbulent flow phenomena from a unique point of view. It is demonstrated, that application of the Riccati-type equations (in both scalar-, and/or matrix forms) - must play a crucial role in the future symmetry analysis of these very general type dissipative structures. Some of the newest research results about influence of helicity on rotational turbulent flows e.g. (Immazio and Minnini, 2013) also justify this concept.

\section{Acknowledgements}

The authors acknowledge support of the Foundation MB/DAAD No. 55731 (2014).

\section{References}

[1] I. Gyarmati, Non-Equilibrium Thermodynamics (Field Theory and Variational Principles), Springer-Verlag, 1970, Berlin - Heidelberg - New York 
[2] R. P.Imazio, P.D Minnini, Passive scalar cascades in rotating helical and non-helical flows, Physica Scripta T155 (2013)014037

[3] L.D. Landau, E. M. LifshitZ, Fluid Mechanics, $2^{\text {nd }}$ Ed., Oxford: ButterworthHeinemann, 2000.

[4] L. D. Landau, E.M. Lifshitz, Statistical Physics 1. $3^{\text {rd }}$ Rev. Ed., Oxford: ButterworthHeinemann, 2000.

[5] Cs. Mészáros, I.Farkas, K. Gottschalk, L. SzÉkely, Á BÁlint. A novel-type solution of the convection-diffusion equation for porous media, $18^{\text {th }}$ International Drying Symposium (IDS 2012) Xiamen, China, 11-15 November 2012

[6] D.S. Mitrinović, Lectures on Differential Equations (Predavanja o diferencijalnim jednainama - in Serbian), Graðevinska Knjiga, 1983, Belgrade

[7] M.I. TRIBELSKII, Short-wavelength instability and transition to chaos in distributed systems with additional symmetry, Physics - Uspekhi 40 (1997) 159 - 180.

[8] T. Vuković, I. MilošEvić, M. DAMnjanović, Molien functions and commensurability of the helicoidal ordering. Physics Letters A 216, (1996) 307 - 312.

[9] M.I. Zelikin, Control Theory and Optimization I. (Homogeneous Spaces and the Riccati Equation in the Calculus of Variations), Springer-Verlag, 2000, Berlin-Heidelberg-New York 